\title{
Supporting Information for Tuplewise Material Representation Based Machine Learning for Accurate Band Gap Prediction
}

\author{
Gyoung S. Na, Seunghun Jang, Yea-Lee Lee*, and Hyunju Chang* \\ Chemical Data-Driven Research Center, Korea Research Institute of Chemical Technology \\ (KRICT), Daejeon, 34114, Korea \\ E-mail: yealee@krict.re.kr; hjchang@krict.re.kr
}


Table S1] shows the selected elemental properties to generate the input node-feature matrix of crystal graph convolutional neural network (CGCNN) and tuplewise graph neural networks (TGNN). For the input edge-feature matrix, we used bond length discretized based on radial basis function kernel (RBF). To generate graph-based representation for CGCNN and TGNN, the inter-atomic bond length cutoff was fixed to $5 \AA$ for all datasets. The tuple generation cutoff of TGNN was set to 3 for PRB-270 dataset [1] and 2 for other datasets. We used Scikit-learn! to implement linear regression and SVR-18. For XGBoost, we employed Py-XGBoost library $\left.\right|^{2}$. We used Python Mendeleev Packag $\rfloor^{3}$ to generate input node-feature matrix and PyTorch Geometric Library to implement CGCNN and TGNN architectures.

Table S1: Selected atomic features and their description.

\begin{tabular}{lll}
\hline Name & Category & Unit \\
\hline Atomic volume & Size & $\mathrm{cm}^{3} / \mathrm{mol}$ \\
Atomic weight & Size & - \\
Covalent radius by Bragg & Size & $\mathrm{pm}$ \\
Fusion heat & Heat & $\mathrm{kJ} / \mathrm{mol}$ \\
Atomic number & Electronic & - \\
Electron affinity & Electronic & $\mathrm{eV}$ \\
First ionization energy & Electronic & $\mathrm{eV}$ \\
Pauling's scale of electronegativity & Electronic & - \\
Period in periodic table & Electronic & - \\
\hline
\end{tabular}

\footnotetext{
${ }^{1}$ https://scikit-learn.org/

${ }^{2}$ https://xgboost.readthedocs.io/en/latest/

${ }^{3}$ https://pypi.org/project/mendeleev/

${ }^{4}$ https://pytorch-geometric.readthedocs.io/en/latest/
} 
Table $\mathrm{S2}$ shows the ranges of the input and target band gaps of the benchmark materials datasets for experimental evaluations. As shown in the table, the input band gaps are frequently underestimated compared with the target band gaps.

Table S2: Range of band gaps for the benchmark datasets.

\begin{tabular}{lll}
\hline Dataset & Range of input band gaps & Range of target band gaps \\
\hline HOIP & {$[1.03,5.34]$} & {$[1.52,6.63]$} \\
C2DB & {$[0,6.45]$} & {$[0,8.63]$} \\
NLHM & {$[0,9.06]$} & {$[0.11,16.03$} \\
PRB-270 & {$[0,9.21]$} & {$[0.36,14.55]$} \\
\hline
\end{tabular}

Figure S1 represents the prediction results by plotting calculated band gap by DFT calculations versus predicted band gap by ML methods. The results are shown for four datasets of HOIP, C2DB, NLHM, and PRB-270 datasets and five ML algorithms. 
(a) HOIP: rPW81-GGA $\rightarrow$ HSE band gap

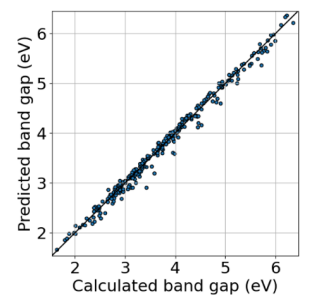

Linear regression

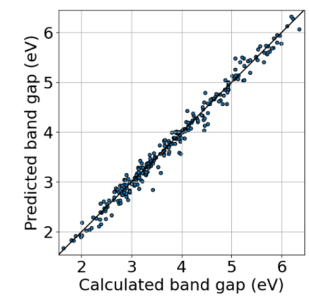

XGBoost

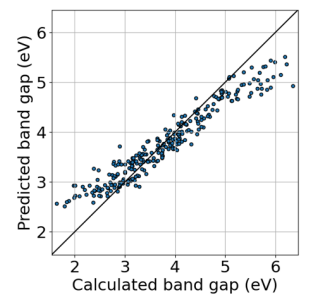

SVR-18

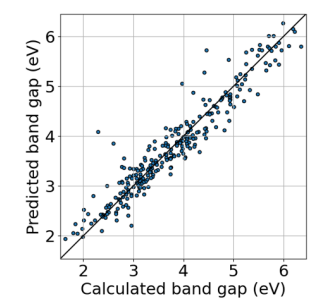

mCGCNN

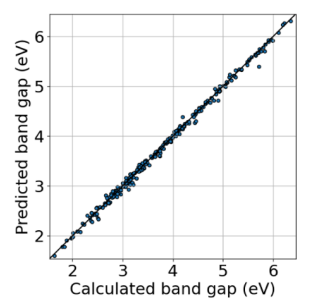

TGNN

(b) C2DB : PBE-GGA $\rightarrow$ HSE band gap

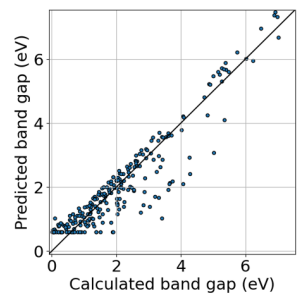

Linear regression

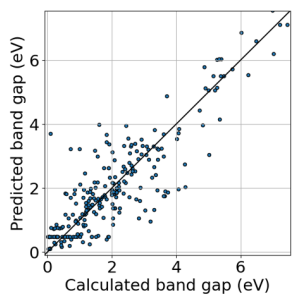

XGBoost

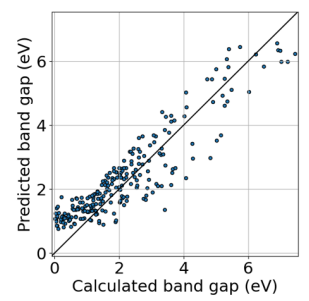

SVR-18

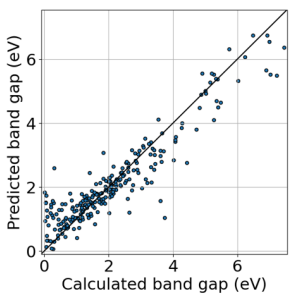

mCGCNN

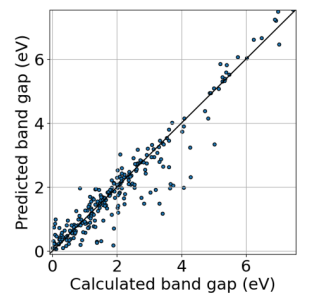

TGNN

(c) NLHM: PBE-GGA $\rightarrow$ GLLB-SC band gap

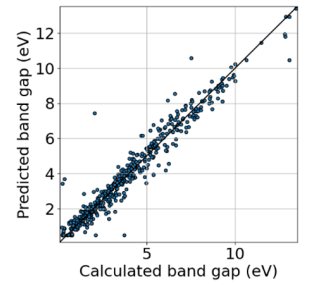

Linear regression

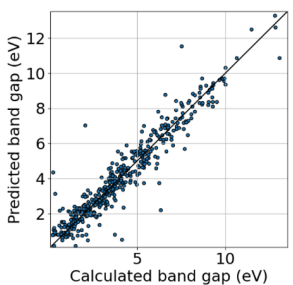

XGBoost

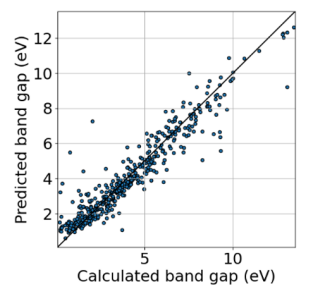

SVR-18

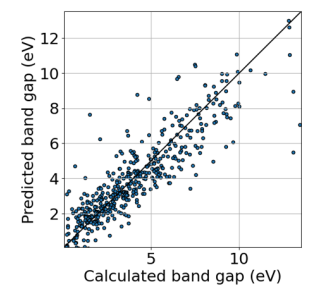

mCGCNN

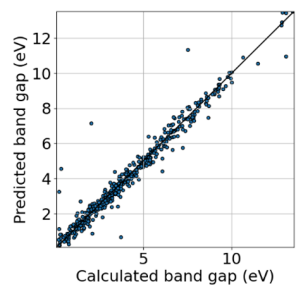

TGNN

(d) PRB-270: PBE-GGA $\rightarrow \mathrm{G}_{0} \mathrm{~W}_{0}$ band gap

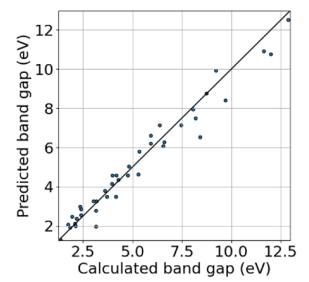

Linear regression

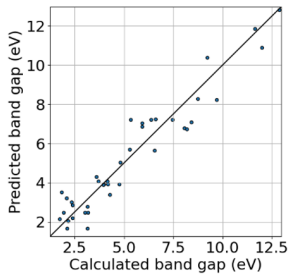

XGBoost

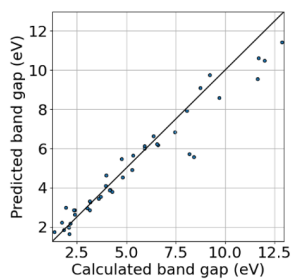

SVR-18

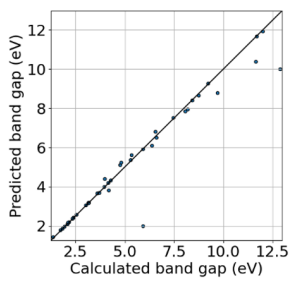

mCGCNN

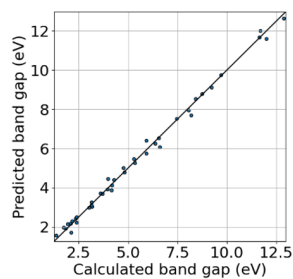

TGNN

Figure S1: Band gap prediction results for (a) HOIP, (b) C2DB, (c) NLHM, and (d) PRB-270 datasets with five different machine-learning methods of linear regression, XGBoost, SVR-18, mCGCNN, and TGNN methods. 
Table S3 shows the validation set for prediction model of TGNN used in Fig. 3 (b) of main paper. The dataset of experimental band gap [2] and corresponding compound identified by the IDs of Materials Project dataset [3] were included.

Table S3: Band gap dataset for validataion. $\mathbf{E}_{g}(\operatorname{Exp}), \mathbf{E}_{g}(\mathrm{PBE})$, and $\mathbf{E}_{g}$ (pred) are experimentally measured band gap, calculated band gap by PBE-GGA functional, and predicted band gap by TGNN, respectively. mp-ID is the ID number of compounds in Materials Project. All band gap data are in $\mathrm{eV}$.

\begin{tabular}{|c|c|c|c|c|c|}
\hline Composition & Space group [2] & $\mathbf{E}_{g}(\operatorname{Exp})[2]$ & mp-ID [3] & $\mathbf{E}_{g}(\mathrm{PBE})[3$ & $\mathbf{E}_{g}($ pred $)$ \\
\hline $\mathrm{Al}_{2} \mathrm{O}_{3}$ & 167 & 8.8 & mp-1143 & 5.85 & 9.44 \\
\hline $\mathrm{AlAs}$ & 216 & 2.23 & mp-2172 & 1.50 & 2.45 \\
\hline $\mathrm{AlN}$ & 186 & 6.13 & mp-661 & 4.04 & 6.29 \\
\hline $\mathrm{AlP}$ & 216 & 2.45 & mp-1550 & 1.63 & 2.76 \\
\hline $\mathrm{AlSb}$ & 216 & 1.68 & mp-2624 & 1.23 & 1.99 \\
\hline BAs & 216 & 1.46 & mp-10044 & 1.20 & 2.08 \\
\hline $\mathrm{BeS}$ & 216 & 5.51 & mp-422 & 3.15 & 4.98 \\
\hline BeTe & 216 & 2.8 & mp-252 & 2.02 & 3.33 \\
\hline $\mathrm{Bi}$ & 166 & 0.01 & mp-23152 & 0.00 & 0.95 \\
\hline $\mathrm{Bi}_{2} \mathrm{Se}_{3}$ & 166 & 0.16 & mp-541837 & 0.54 & 1.86 \\
\hline $\mathrm{Bi}_{2} \mathrm{Te}_{3}$ & 166 & 0.13 & mp-34202 & 0.53 & 1.66 \\
\hline $\mathrm{BN}$ & 216 & 6.22 & mp-1639 & 4.45 & 6.71 \\
\hline $\mathrm{BP}$ & 216 & 2.4 & mp-1479 & 1.24 & 2.29 \\
\hline $\mathrm{C}$ & 227 & 5.41 & mp-66 & 4.11 & 6.44 \\
\hline $\mathrm{CaO}$ & 225 & 7.7 & mp-2605 & 3.63 & 6.53 \\
\hline CaSe & 225 & 3.85 & mp-1415 & 2.08 & 3.91 \\
\hline CaSe & 225 & 3.85 & mp-1415 & 2.08 & 3.91 \\
\hline $\mathrm{CdGa}_{2} \mathrm{Se}_{4}$ & 82 & 2.33 & mp-3772 & 1.35 & 2.99 \\
\hline $\mathrm{CdIn}_{2} \mathrm{Se}_{4}$ & 82 & 1.82 & mp-22304 & 0.95 & 2.27 \\
\hline $\mathrm{CdO}$ & 225 & 0.84 & mp-1132 & 0.00 & 1.55 \\
\hline $\mathrm{CdS}$ & 216 & 2.55 & mp-2469 & 1.05 & 2.53 \\
\hline $\mathrm{CdTe}$ & 216 & 1.92 & mp-406 & 0.58 & 1.86 \\
\hline $\mathrm{CoO}$ & 225 & 2.6 & mp-19079 & 0.00 & 2.02 \\
\hline $\mathrm{CuAlO}_{2}$ & 166 & 3 & mp-3748 & 1.80 & 4.32 \\
\hline $\mathrm{CuGaO}_{2}$ & 166 & 3.6 & mp-4280 & 0.73 & 2.99 \\
\hline $\mathrm{CuInO}_{2}$ & 166 & 3.9 & mp-20930 & 0.24 & 1.82 \\
\hline $\mathrm{Fe}_{2} \mathrm{O}_{3}$ & 167 & 2 & mp-19770 & 1.53 & 4.20 \\
\hline
\end{tabular}

(continued) 


\begin{tabular}{|c|c|c|c|c|c|}
\hline Composition & Space group [2] & $\mathbf{E}_{g}(\mathbf{E x p})[2$ & mp-ID 3 & $\mathbf{E}_{g}(\mathrm{PBE})[3$ & $\mathbf{E}_{g}($ pred $)$ \\
\hline GaAs & 216 & 1.52 & $\mathrm{mp}-2534$ & 0.19 & 1.54 \\
\hline $\mathrm{GaN}$ & 216 & 3.3 & mp-830 & 1.56 & 3.24 \\
\hline $\mathrm{GaP}$ & 216 & 2.35 & mp-2490 & 1.59 & 2.60 \\
\hline $\mathrm{GaSb}$ & 216 & 0.73 & mp-1156 & 0.00 & 0.99 \\
\hline $\mathrm{Ge}$ & 227 & 0.66 & mp-32 & 0.00 & 0.95 \\
\hline $\mathrm{HgTe}$ & 216 & 0.14 & mp-2730 & 0.00 & 0.96 \\
\hline $\mathrm{In}_{2} \mathrm{O}_{3}$ & 167 & 3.02 & mp-22323 & 0.96 & 3.20 \\
\hline InAs & 216 & 0.35 & mp-20305 & 0.00 & 0.96 \\
\hline $\operatorname{InN}$ & 186 & 0.69 & mp-22205 & 0.00 & 0.96 \\
\hline $\mathrm{InP}$ & 216 & 1.42 & mp-20351 & 0.47 & 1.38 \\
\hline $\mathrm{InSb}$ & 216 & 0.18 & mp-20012 & 0.00 & 0.95 \\
\hline $\mathrm{KCl}$ & 225 & 8.5 & mp-23193 & 5.03 & 8.51 \\
\hline $\mathrm{KF}$ & 225 & 10.9 & $\mathrm{mp}-463$ & 6.00 & 10.47 \\
\hline $\mathrm{LiF}$ & 225 & 14.2 & mp-1138 & 8.72 & 14.21 \\
\hline $\mathrm{MgO}$ & 225 & 7.83 & mp-1265 & 4.44 & 7.86 \\
\hline $\mathrm{MgS}$ & 225 & 5.4 & mp-1315 & 2.76 & 4.87 \\
\hline $\mathrm{MnO}$ & 225 & 3.7 & mp-19006 & 1.95 & 4.59 \\
\hline $\mathrm{MoS}_{2}$ & 194 & 1.29 & mp-2815 & 1.23 & 3.09 \\
\hline $\mathrm{NaCl}$ & 225 & 9 & mp-22862 & 5.00 & 8.83 \\
\hline $\mathrm{NaI}$ & 225 & 5.9 & mp-23268 & 3.53 & 6.07 \\
\hline $\mathrm{NiO}$ & 225 & 3.7 & mp-19009 & 2.30 & 5.03 \\
\hline $\mathrm{PbTe}$ & 225 & 0.31 & mp-19717 & 0.81 & 1.77 \\
\hline $\mathrm{RbCl}$ & 225 & 8.3 & mp-23295 & 4.84 & 8.43 \\
\hline $\mathrm{RbI}$ & 225 & 5.83 & mp-22903 & 3.78 & 6.57 \\
\hline $\mathrm{Sb}_{2} \mathrm{Te}_{3}$ & 166 & 0.23 & mp-1201 & 0.29 & 1.46 \\
\hline $\mathrm{Si}$ & 227 & 1.12 & mp-149 & 0.61 & 1.30 \\
\hline $\mathrm{SiC}$ & 216 & 2.42 & mp-8062 & 1.37 & 2.58 \\
\hline $\mathrm{SiO}_{2}$ & 92 & 8.9 & mp-6945 & 5.55 & 9.46 \\
\hline $\mathrm{SiO}_{2}$ & 154 & 9.65 & mp-7000 & 5.72 & 9.62 \\
\hline $\mathrm{SnSe}$ & 62 & 0.86 & mp-691 & 0.52 & 1.65 \\
\hline $\mathrm{TiO}_{2}$ & 141 & 3.4 & mp-390 & 2.05 & 5.07 \\
\hline $\mathrm{TiO}_{2}$ & 136 & 3.3 & mp-2657 & 1.78 & 4.72 \\
\hline $\mathrm{VO}_{2}$ & 136 & 0.6 & mp-19094 & 0.86 & 3.54 \\
\hline $\mathrm{WO}_{3}$ & 14 & 2.9 & mp-619461 & 1.37 & 4.46 \\
\hline $\mathrm{Zn}_{3} \mathrm{~N}_{2}$ & 206 & 1 & mp-9460 & 0.00 & 1.42 \\
\hline $\mathrm{ZnIn}_{2} \mathrm{Se}_{4}$ & 82 & 2 & mp-22607 & 0.99 & 2.43 \\
\hline $\mathrm{ZnO}$ & 186 & 3.44 & mp-2133 & 0.73 & 3.11 \\
\hline $\mathrm{ZnS}$ & 216 & 3.66 & mp-10695 & 2.02 & 3.96 \\
\hline $\mathrm{ZnSe}$ & 216 & 2.7 & mp-1190 & 1.17 & 3.01 \\
\hline $\mathrm{ZnTe}$ & 216 & 2.38 & $\mathrm{mp}-2176$ & 1.03 & 2.75 \\
\hline
\end{tabular}




\section{References}

(1) Lee, J.; Seko, A.; Shitara, K.; Nakayama, K.; Tanaka, I. Prediction model of band gap for inorganic compounds by combination of density functional theory calculations and machine learning techniques. Phys. Rev. B 2016, 93, 115104.

(2) Morales-García,; Ángel,; Valero,; Rosendo,; Illas, F. An Empirical, yet Practical Way To Predict the Band Gap in Solids by Using Density Functional Band Structure Calculations. J. Phys. Chem. C 2017, 121, 18862-18866.

(3) Jain, A.; Ong, S. P.; Hautier, G.; Chen, W.; Richards, W. D.; Dacek, S.; Cholia, S.; Gunter, D.; Skinner, D.; Ceder, G. et al. Commentary: The Materials Project: A materials genome approach to accelerating materials innovation. APL Materials 2013, 1, 011002. 